\title{
Brucella and Coxiella; if you don't look, you don't find
}

\author{
Authors: Jonathan R Lambourne ${ }^{A}$ and Tim Brooks ${ }^{B}$
}

Brucella and Coxiella are similar; both are obligate intracellular, zoonotic pathogens with a broad geographic distribution. Infection in animals is usually asymptomatic, but causes fetal loss and therefore has significant economic impact. Human infection may be asymptomatic or give rise to either organ-specific or multi-system disease. Organism culture is challenging for Coxiella and can lack sensitivity for Brucella. Therefore, infection is most commonly diagnosed by serology, but this may be negative in early infection and serology results may be challenging to interpret. Both Brucella and Coxiella are typically susceptible to a wide range of antimicrobials, but long courses may be needed.

\section{Coxiella}

Coxiella burnetii is the cause of Q fever, a term first used in 1983 during the investigation of a cluster of febrile Australian meat workers. ${ }^{1}$ Infection typically follows inhalation or ingestion of spores, which are able to survive in soil, dairy produce and water for many months. Direct animal contact is not required as spores may be carried long distances by the wind. Coxiella is endemic in most countries. In Southern Europe and North Africa it is the causative agent in up to $10 \%$ of patients admitted with a febrile illness, ${ }^{2}$ and seroprevalence rates of over $30 \%$ have been reported in some countries, including Holland. ${ }^{3,4}$ Asymptomatic or mild infection is probably the norm following inoculation. What determines whether initial exposure is followed by eradication or chronic carriage is as yet unknown. Acute infection presents following an incubation period of 2-5 weeks and often manifests as an undifferentiated febrile illness, a respiratory illness with cough and variable radiological findings, ${ }^{5}$ or as an aseptic meningitis, usually with a normal cerebrospinal fluid (CSF) white cell count but raised protein and is often accompanied by hepatitis. ${ }^{6}$ Chronic infection can result in endocarditis, hepatitis, osteomyelitis and central nervous system (CNS) infection. Coxiella endocarditis usually affects abnormal or prosthetic valves and is often complicated by arterial emboli and immune

Authors: ${ }^{A}$ specialty trainee in infectious diseases and microbiology, Hospital for Tropical Diseases, University College London Hospitals NHS Trust, London, UK; ${ }^{B}$ consultant microbiologist and clinical services director of the Rare and Imported Pathogens Laboratory, Public Health England, Salisbury, UK complex-mediated skin and renal disease. However fever may be absent. ${ }^{7}$ Reactivation of latent infection is associated with impaired cell-mediated immunity, including that associated with pregnancy. ${ }^{8}$

Coxiella cannot be cultured using routine lab methods. Serology is the mainstay of diagnosis, ${ }^{9,10}$ but may take 2-6 weeks to become positive, up to $60 \%$ of acutely infected patients have negative initial serology. ${ }^{11}$ Polymerase chain reaction (PCR) assays can rapidly and reliably diagnose acute Q fever prior to development of a detectable antibody response. ${ }^{12}$ During infection, C burnetii varies its lipopolysaccharide (LPS) coat, the primary antigenic target. ${ }^{13}$ Phase II LPS is produced first, switching to phase I LPS several weeks to months later. ${ }^{10}$ Acute $\mathrm{Q}$ is diagnosed by a positive PCR or by a four-fold increase in phase II IgG antibody titer between acute and convalescent paired sera, and is suggested by either phase II IgM $\geq 1: 50$ and/or phase II IgG $\geq 1: 200$. ${ }^{6,10}$ Chronic $Q$ is diagnosed by phase $\operatorname{IgG} \geq 1: 800$ and suggested by phase I IgG $\geq 1: 128$ and $<1: 800 .{ }^{6}$ In chronic infection, IgM is typically negative but titres may remain elevated for 12 months.

Coxiella are typically susceptible to a range of antimicrobials including tetracyclines, rifampicin, chloramphenicol, fluroquinolones and macrolides. Hydroxychloroquine alkalinises phagolysosomes, in which Coxiella resides, ${ }^{13,14}$ and may help increase the bactericidal effect of some of these agents. The ideal agent(s) and duration vary with the disease manifestation; acute infection usually requires $2-3$ weeks, whereas chronic infection may require many months of treatment, guided by clinical progress and lab investigations such as Coxiella titres, erythrocyte sedimentation rate (ESR) and haemoglobin. ${ }^{6}$ A transthoracic echocardiogram should be considered for all patients diagnosed with acute $\mathrm{Q}$, as patients with valvular abnormalities are predisposed to chronic infection, and should undergo regular serological follow up. ${ }^{15}$

\section{Brucella}

In 1887, Dr David Bruce described the causal relationship between Brucella species and the disease 'Malta fever'. Human infection occurs following inhalational or mucosal contact with animal blood and body fluids, or ingestion of infected unpasteurised milk, cheese and meat. Brucella may be viable for 48 hours in milk, weeks in frozen meat and 3 months in unpasteurised cheese. ${ }^{16}$ A number of Brucella species have been reported to cause human disease, including $B$ melitensis, $B$ abortus, $B$ suis and $B$ canis, with $B$ melitensis and $B$ suis associated with more severe disease. There is some speciesreservoir specificity, for example, $B$ melitensis affects mainly 
ungulates (goats, sheep and camels) whereas $B$ abortus mainly infects horses and ruminants. ${ }^{17}$ Brucella is the most common bacterial zoonosis worldwide, with at least 500,000 new cases reported each year. Brucella infection causes disease worldwide, especially in Arabia, the Mediterranean, Latin America and Central Asia. ${ }^{17}$ It has been reported that, in Kuwait, Brucella infection accounts for $10 \%$ of acute medical admissions. ${ }^{18}$

Following inoculation, many individuals remain asymptomatic. Clinical disease may be acute ( $<1$ month), subacute (1-6 months) or chronic ( $>6$ months) and may present with systemic upset or disease limited to a single organ system..$^{18}$ Common acute manifestations include an undifferentiated febrile illnesses, meningitis, encephalitis, septic arthritis and epididymo-orchitis. Chronic manifestations include systemic upset alone and/ or with localised infection such as spondylitis, uveitis and endocarditis. Brucella may cause an 'undulant' fever, typically occurring in the late afternoon, which may be associated with focal sweating, which may have a strong mouldy smell.

Brucella grows on standard lab media, but detection requires prolonged incubation due to the organisms' slow doubling time. Culture sensitivity varies from $15 \%$ to $90 \%$ depending on disease stage, sample type and culture media used. ${ }^{19}$ Sensitivity is highest in early infection and when bone marrow samples are cultured. PCR has shown promise, but there are no standardised methods. Serology is the mainstay of diagnosis, and has a sensitivity ranging from $65 \%$ to $95 \%$, depending on disease stage and technique(s) used. The Public Health England Brucella reference unit performs four assays on all samples; microscopic agglutination (MAG), complement fixation (CF), and IgG and IgM-specific ELISAs. ${ }^{20}$ Brucella infection is suggested by the following; MAG titre $\geq 1: 160$, CF titre $>1: 4$ and/or enzyme-linked immunosorbent assay (ELISA) titre $\geq 1: 20$. Comparing IgG and IgM titres can help differentiate acute from chronic infection, although elevated IgM titres are present for 12 months in $50 \%$ of cases.

Brucella species are typically susceptible to a range of antibiotics, including tetracyclines, rifampicin, aminoglycosides, ceftriaxone and cotrimoxazole. Its intracellular sanctuary site and slow doubling time warrants courses of at least six weeks. Longer treatment and/or combination therapy may be used for more 'complicated' infections, such as spondylitis, neurobrucellosis, endocarditis. ${ }^{21}$ Relapse occurs in $5-15 \%$ of

\section{Key points}

Brucella and Coxiella are zoonoses with a wide geographic distribution

Human infection may be asymptomatic, organ-specific or multi-system

Serological tests may be negative in early infection

Long courses of antimicrobials may be required for effective treatment

KEYWORDS: Brucella, Coxiella, zoonosis, diagnosis, fever cases, typically due to inadequate adherence, dose or duration. Relapse due to antibiotic resistance is rare.

\section{References}

1 Derrick EH. "Q" fever, a new fever entity: clinical features, diagnosis and laboratory investigation. Rev Infect Dis 1983;5:790-800.

2 Vanderburg S, Rubach MP, Halliday JE, Cleaveland S, Reddy EA, Crump JA. Epidemiology of Coxiella burnetii infection in Africa: a OneHealth systematic review. PLoS Negl Trop Dis 2014;8:e2787.

3 Richardus JH, Donkers A, Dumas AM et al. Q fever in the Netherlands: a sero-epidemiological survey among human population groups from 1968 to 1983. Epidemiol Infect 1987;98:211-9.

4 Mak DB, Fry DF, Bulsara MK. Prevalence of markers of Q fever exposure in the Kimberley, Western Australia. Commun Dis Intell Q Rep 2003;27:267-71.

5 Marrie TJ. Coxiella burnetii pneumonia. Eur Respir J 2003;21:713-9.

6 Anderson A, Bijlmer H, Fournier PE et al. Diagnosis and management of Q fever - United States, 2013: recommendations from CDC and the Q Fever Working Group. MMWR Recomm Rep 2013;62:1-30.

7 Marrie TJ, Raoult D. Q fever - a review and issues for the next century. Int J Antimicrob Agents 1997;8:145-61.

8 Langley JM, Marrie TJ, Leblanc JC, Almudevar A, Resch L, Raoult D. Coxiella burnetii seropositivity in parturient women is associated with adverse pregnancy outcomes. Am J Obstet Gynecol 2003;189:228-32.

9 Raoult D, Marrie T. Q fever. Clin Infect Dis 1995;20:489-95; quiz 96.

10 Fournier PE, Marrie TJ, Raoult D. Diagnosis of Q fever. J Clin Microbiol 1998;36:1823-34.

11 Fournier PE, Raoult D. Comparison of PCR and serology assays for early diagnosis of acute Q fever. J Clin Microbiol 2003;41:5094-8.

12 Schneeberger PM, Hermans MH, van Hannen EJ, Schellekens JJ, Leenders AC, Wever PC. Real-time PCR with serum samples is indispensable for early diagnosis of acute $\mathrm{Q}$ fever. Clin Vaccine Immunol 2010;17:286-90.

13 Voth DE, Heinzen RA. Lounging in a lysosome: the intracellular lifestyle of Coxiella burnetii. Cell Microbiol 2007;9:829-40.

14 Flannagan RS, Cosio G, Grinstein S. Antimicrobial mechanisms of phagocytes and bacterial evasion strategies. Nat Rev Microbiol 2009;7:355-66.

15 Wielders CC, Morroy G, Wever PC, Coutinho RA, Schneeberger PM, van der Hoek W. Strategies for early detection of chronic Q-fever: a systematic review. Eur J Clin Invest 2013;43:616-39.

16 Young EJ, Suvannoparrat U. Brucellosis outbreak attributed to ingestion of umpasteurized goat cheese. Arch Intern Med 1975;135:240-3.

17 Franco MP, Mulder M, Gilman RH, Smits HL. Human brucellosis. Lancet Infect Dis 2007;7:775-86.

18 Lulu AR, Araj GF, Khateeb MI, Mustafa MY, Yusuf AR, Fenech FF. Human brucellosis in Kuwait: a prospective study of 400 cases. Q J Med 1988;66:39-54.

19 Memish Z, Mah MW, Al Mahmoud S, Al Shaalan M, Khan MY. Brucella bacteraemia: clinical and laboratory observations in 160 patients. J Infect 2000;40:59-63.

20 Sharma R, Chisnall C, Cooke RP. Evaluation of in-house and commercial immunoassays for the sero-diagnosis of brucellosis in a non-endemic low prevalence population. J Infect 2008;56:108-13.

21 Corbel MJ, Elberg SS, Cosivi O. Brucellosis in humans and animals. Geneva: World Health Organization, 2006.

Address for correspondence: Dr J Lambourne, Rare and Imported Pathogens Laboratory, Public Health England, Porton Down, Salisbury, SP4 OJG, UK.

Email: jonathan.lambourne@nhs.net 